\title{
A criterion for nonlinear wave stability
}

\author{
By K. HASSELMANN \\ University of Hamburg
}

(Received 3 July 1967)

Nonlinear resonant wave triads composed of one finite and two infinitesimal components are unstable for sum interactions and neutrally stable for difference interactions. A similar criterion holds for tertiary interactions.

In the preceding paper Davis \& Acrivos (1967) have discussed conditions under which resonant interactions between an internal gravity-wave mode and two infinitesimal internal modes lead to an exponential growth of the infinitesimal components. The authors' results for undamped modes can be deduced from the following simple stability criterion, which applies to all conservative coupledmode systems independent of the details of the coupling. The nonlinear coupling between two infinitesimal components 1 and 2 and a finite component 0 whose wave-numbers and frequencies satisfy the resonant-interaction conditions

$$
\mathbf{k}_{1} \pm \mathbf{k}_{\mathbf{2}}=\mathbf{k}_{0}, \quad \omega_{1} \pm \omega_{2}=\omega_{0}
$$

is unstable for the sum interaction and neutrally stable for the difference interaction. In equation (1), $\mathbf{k}_{j}$ points in the direction of wave propagation and $\omega_{j}>0$.

The equations of motion of a conservative, coupled-mode system may be written in the form (Hasselmann 1966, equation (1.13))

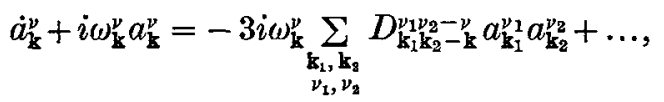

where $a_{\mathrm{k}}^{v}$ denotes the time-dependent factor of the suitably normalized normal mode $\nu, \omega_{\mathrm{k}}^{\nu}$ is the mode eigenfrequency and $D_{\mathbf{k}_{1} k_{2} \mathbb{K}_{3}}^{\nu_{2} \nu_{3}}$ is a symmetrical coupling coefficient which is non-zero only for $\mathbf{k}_{1}+\mathbf{k}_{\mathbf{2}}+\mathbf{k}_{3}=0$. Reality requirements yield the relations

$$
\begin{aligned}
& a_{\mathrm{k}}^{\nu}=\left(a_{-\mathrm{k}}^{-y}\right)^{*}, \\
& D_{\mathbf{k}_{1} \mathbf{k}_{2} \mathbf{k}_{3}}^{\nu_{1} \nu_{3} \nu_{3}}=\left(D_{-\mathbf{k}_{1}-\mathbf{k}_{2}-\mathbf{k}_{3}}^{-\nu_{1}-\nu_{2}-\nu_{3}}\right) * \text {. }
\end{aligned}
$$

The mode indices $\nu$ and eigenfrequencies $\omega_{\mathrm{k}}^{\nu}$ take two signs, corresponding to opposite propagation directions. Thus if the spatial dependence is given by $e^{i \mathbf{k} . \mathbf{x}}$, $\nu>0$ is associated with a linear wave solution $a_{\mathrm{k}}^{\nu}=A \exp \left\{-i \omega_{\mathrm{k}}^{\nu} t\right\}, A=$ const., $\omega_{\mathrm{k}}^{\nu}>0$, which propagates in the positive $\mathbf{k}$ direction; $\nu<0$ corresponds to a wave component of negative frequency propagating in the opposite direction.

Consider now the interaction between three discrete modes $v_{1}, \nu_{\mathrm{a}}, \nu_{0}\left(v_{j}<0\right)$ whose wave-numbers and frequencies satisfy the resonance-interaction conditions

$$
\mathbf{k}_{1}+\mathbf{k}_{\mathbf{2}}=\mathbf{k}_{0}, \quad \omega_{1}+\omega_{2}=\omega_{0}
$$


where $\omega_{j} \equiv \omega_{\mathbf{k}_{j}}^{\boldsymbol{y}_{j}} \gtrless 0$. (The more easily interpretable sign convention of equations (1) is obtained by changing the signs of $\mathbf{k}_{j}$ and $\omega_{j}$ for negative indices $\nu_{j}$.) Writing $a_{\mathbf{k} j}^{\nu j}=A_{j}(t) \exp \left\{-i \omega_{j} t\right\}$, where $A_{j}$ is a slowly varying amplitude, equation $(\mathrm{H} 1.13)$ yields

$$
\left.\begin{array}{l}
\dot{A}_{1}=-3 i \omega_{1} D^{*} A_{0} A_{2}^{*}, \\
A_{2}^{*}=3 i \omega_{2} D A_{01}^{*} A_{1} \quad\left(D \equiv D_{\mathbf{k}_{1} \mathbf{k}_{2}-\mathbf{k}_{0}}^{\nu_{1} \nu_{2}-\nu_{0}}\right) .
\end{array}\right\}
$$

A third equation shows that $\dot{A}_{0}=O\left(A_{1} A_{2}\right)$, so that, for $\left|A_{1}\right|,\left|A_{2}\right| \ll\left|A_{0}\right|, A_{0}$ can be regarded as constant.

The eigenvalues of (2) are

$$
\lambda= \pm 3\left(\omega_{1} \omega_{2}\right)^{\frac{1}{2}}|D|\left|A_{0}\right| . \quad \text { Q.E.D. }
$$

For $\omega_{1}=\omega_{2}=\frac{1}{2} \omega_{0}$, the criterion yields the well-known case of subharmonic parametric instability considered, for example, by Kelly (1965). Kelly's (1967) results for nonlinear resonant interactions in a shear layer are also in accordance with the stability criterion (Kelly's case A is a sum interaction, while his case B is a difference interaction). However, it should be noted that the criterion is strictly applicable only to conservative wave-wave interactions, and should therefore be applied with caution to wave components coupled to an external energy source, such as Tollmien-Schlichting waves.

Under normal laboratory conditions, the background disturbances are probably better represented by a continuous spectrum than two discrete lines. However, the continuous case follows by straightforward superposition, since the perturbation equations are linear in the infinitesimal components and all interacting infinitesimal pairs are mutually distinct. The growth of the infinitesimal components is limited to a lower-dimensional subspace of the continuum by the resonant interaction conditions. (For this reason, the continuum energy transfer formalism (Hasselmann 1966) is not applicable to second-order interactions of a discrete line with a continuum.)

In the case of third-order interactions, involving a finite component and three infinitesimal components, the perturbation equations are quadratic in the infinitesimal components, so that the eigenvalue approach and the superposition principle fail. Nogeneral stability criterion appears to exist. The stability depends on the details of the disturbances. In the case of a discrete gravity-wave component superimposed on a continuum, the stability is determined by the shape of the spectrum. Inspection of the general transfer equation (2.5) (Hasselmann 1966) shows that unstable distributions always exist. (For finite disturbances, all distributions are unstable, as follows from the irreversible tendency of all distributions to a white spectrum. The same applies to second-order interactions of a finite-width line with a finite-energy background.)

For third-order interaction involving only two infinitesimal components 1 and 2 and a finite component 0 that enters twice in the interaction (so that the resonance conditions are identical with (1), except that $\mathbf{k}_{0}, \omega_{0}$ are replaced by $\left.2 \mathbf{k}_{0}, 2 \omega_{0}\right)$, one obtains the same stability criterion as before in the secondorder case. This again follows alone from the symmetry of the coupling coefficients and is independent of the details of the coupling. 
In practice, the interaction $(1) \pm(2)=2(0)$ never occurs alone but is accompanied by the interactions $(1)-(1)=(0)-(0)$ and $(2)-(2)=(0)-(0)$. Since the coupling coefficients $D_{\mathbf{k}_{0}-\mathbf{k}_{0} \mathbf{k}_{j}-\mathbf{k}_{j}}^{\omega_{0} \omega_{0}}, j=1,2$, are real (equation $\mathrm{H}_{1.12}$ ), the eigenvalues of these interactions are imaginary, $\lambda_{j}=i \Omega_{j}$, say. This corresponds to a change in the phase velocities, as discussed for the case of gravity waves by Longuet-Higgins \& Phillips (1962). The superposition of all three interactions then yields eigenvalues

$$
\lambda= \pm\left[\lambda_{12}^{2}-\left(\frac{\Omega_{1}+\Omega_{2}}{2}\right)^{2}\right]^{\frac{1}{2}}
$$

where $\lambda_{12}$ represents the eigenvalue of the interaction $(1) \pm(2)=2(0)$.

Thus the phase-velocity perturbations appear to have a stabilising influence. However, the frequency shifts $\Omega_{j}$ can normally be neutralised by considering slightly detuned interactions for which $\omega_{1}+\omega_{2}=2 \omega_{0}+\Omega_{1}+\Omega_{2}$, so that their net effect is simply to slightly distort the region of instability in the wave-number plane. An exception are the singular points for which the frequency sum $\omega_{1}+\omega_{2}$ is stationary with respect to wave-number variations (e.g. $\omega_{1}=\omega_{2}=\omega_{0}$ ). Here the sign of the frequency detuning is not disposable, and the stability depends on the details of the coupling coefficients.

An application of the theorem are tertiary gravity wave interactions, which are of the sum type and therefore unstable. (The instability of gravity waves was first found by Benjamin \& Feir (1967), Benjamin (1967) and Witham (1966, 1967). Their analyses applied to side-band interactions close to the singular point $\omega_{1}=\omega_{2}=\omega_{0}$ and thus did involve in this case detailed properties of the coupling.)

\section{REFERENCES}

BenJamin, T. B. 1967 Instability of period wave trains in nonlinear dispersive systems. Proc. Roy. Soc. A 299, 59-75.

Benjualin, T. B. \& FEIR, J. E. 1967 The disintegration of wave trains on deep water. Part 1. Theory. J. Fluid Mech. 27, 417-30. Part 2. Experiments. J. Fluid Mech. (to appear).

Davis, R. E. \& Acrivos, A. 1967 The stability of oscillatory internal waves. J. Fluid Mech. 30, 723.

HasselmanN, K. 1966 Feynman diagrams and interaction rules of wave-wave scattering processes. Rev. Geophys. 4, 1-32.

KELLY, R. E. 1965 The stability of an unsteady Kelvin-Helmholtz flow. J. Fluid Mech. 22, 547-60.

KELLY, R. E. 1967 On the stability of an inviscid shear layer which is periodic in space and time. J. Fluid Mech. 27, 657-89.

Longuet-Higgins, M. S. \& Phillips, O. M. 1962 Phase velocity effects in tertiary wave interactions. $J$. Fluid Mech. 12, 333-336.

Whitham, G. B. 1966 Nonlinear dispersion of water waves. J. Fluid Mech. 27, 399-412.

Whitham, G. B. 1967 Variational methods and applications to water waves. Proc. Roy. Soc. A 299, 6-25. 